\title{
Bibliographic catalogue of stellar radial velocities: $(1991-1994)^{\star}$
}

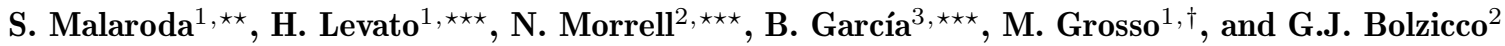 \\ 1 Complejo Astronómico El Leoncito (CASLEO), Casilla de Correo 467, 5400 San Juan, Argentina \\ 2 Facultad de Ciencias Astronómicas y Geofísicas (FCAGLP), Universidad Nacional de La Plata, Paseo del Bosque, Prov. de \\ Buenos Aires, Argentina \\ 3 Centro Regional de Investigaciones Científicas y Técnicas, CRICYT, Mendoza, Argentina
}

Received November 18; accepted December 9, 1999

\begin{abstract}
We present a bibliographic catalogue of stellar radial velocities with 13359 entries for stars in the galaxy and the Magellanic Clouds, obtained from the issues of several journals published in the period 1991-1994. We have tried to follow the structure of previous compilations although we have relied on data retrived from the SIMBAD database.
\end{abstract}

Key words: catalogues — stars: kinematics — techniques: radial velocities

\section{Introduction}

The present catalogue is the first compiled at the Complejo Astronómico El Leoncito and it is intended to be a continuation of previous compilations of bibliographies of stellar radial velocities. The first one was done by Abt \& Biggs (1972), who included the bibliography published before 1970. M. Barbier continued with the cataloguing for the period 1971-1990. She published the bibliographic references in three separate catalogues,

Send offprint requests to: H. Levato,

e-mail: levato@infovia.com.ar

* Catalogue available at the CDS via anonymous ftp to cdsarc.u-strasbg.fr (130.79.128.5) or via http://cdsweb.ustrasbg.fr/Abstract.html

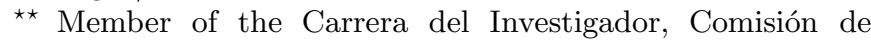
Investigaciones Científicas de la Provincia de Buenos Aires, Argentina.

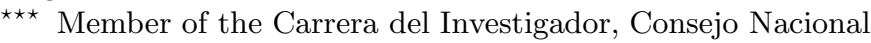
de Investigaciones Científicas y Técnicas, CONICET, Argentina.

$\dagger$ Member of the Carrera del Personal de Apoyo del Consejo Nacional de Investigaciones Científicas y Técnicas, CONICET, Argentina. covering 5 years each (see Barbier \& Petit 1986; Barbier \& Petit 1990; and Barbier et al. 1994). These three catalogues include more than 45000 entries.

We are presenting in the present catalogue the bibliographic references of stellar radial velocities published between 1991 and 1994. The data for the period 1995-1998 will come soon, and we hope to maintain the compilation with yearly updates. The catalogue can be downloaded via ftp from the WEB page of the Complejo Astronómico El Leoncito (www.casleo.secyt.gov.ar). In the future it will be possible to download this catalogue from the CDS database (Strasbourg).

\section{Description of the catalogue}

We have revised the issues of the journals listed in Table 11, for the period 1991-1994. It is not a complete list of all journals devoted to astronomical literature, but they are the most important ones and those available at our libraries.

We prepared a plain ASCII file with the object identifications as provided by the authors. This file was transferred to the SIMBAD ${ }^{1}$ database, and, using the proper formats and filters, we have retrived the identification for each object according to the rules of SIMBAD (Egret 1996); see also Lortet et al. (1986). In the same way we have also retrived: the J2000 coordinates, the $V$ magnitude, and the spectral classification for each object.

The different columns of the catalogue are described as follows:

Column 1 (bytes 1 to 30): Identification. We have preferred for identification the HD, CD or CPD numbers,

\footnotetext{
${ }^{1}$ Operated by the CDS at Strasbourg, France.
} 
Table 1. Revised journals

\begin{tabular}{ll}
\hline Journal & Abbreviation \\
\hline Acta Astronomica & AA \\
Astronomy \& Astrophysics & AAP \\
Astronomy and Astrophysics Suppl. Series & AAPS \\
Astronomical Journal & AJ \\
Astrophysical Journal & APJ \\
Astrophysical Journal Suppl. Series & APJS \\
Bulletin of the Astronomical Institutes of Czechoslovakia & BAIC \\
International Astronomical Union Symposium & IAUS \\
Information Bulletin on Variable Stars & IBVS \\
The Messenger & MES \\
Monthly Notices of the Royal Astronomical Society & MN \\
Monthly Notices of the Astronomical Society of South Africa & MNAS \\
Memorie della Società Astronomica Italiana & MSAI \\
The Observatory & OBS \\
Publications of the Astronomical Society of Japan & PASJ \\
Publications of the Astronomical Society of the Pacific & PASP \\
Journal of the Royal Astronomical Society of Canada & RASC \\
Revista Mexicana de Astronomía y Astrofísica & RMAA \\
Soviet Astronomy & SA \\
Vistas in Astronomy & VA \\
\hline
\end{tabular}

in that order. Stars in clusters are indicated with the cluster's name and the number of the stars according to the primary identification paper for that particular cluster, found either at SIMBAD or Mermilliod's database (which was accessed through the WEB of the Geneva Observatory).

Column 2 (bytes 31 to 37): Right ascension for J2000.0. It is given to the tenth of a minute and has been retrived from SIMBAD.

Column 3 (bytes 44 to 49): Declination for J2000.0. It is given to the minute and has been retrived from SIMBAD.

Column 4 (bytes 56 to 63): Visual magnitude. It has been retrived from SIMBAD.

Column 5 (bytes 65 to 76 ): Spectral type and luminosity class. They have been retrived from the SIMBAD database. In some cases, when the number of symbols is large, the spectral type has been truncated.

Column 6 (bytes 83 to 89 ): Radial velocity. For each object we have included the average radial velocity provided by the author. If the number of radial velocity measurements is more than 1 , and an average has not been calculated by the author, we have not included any radial velocity at all. If the star is a spectroscopic binary, for which an orbit has been computed, then the systemic radial velocity is quoted in this column.

Column 7 (bytes 98 to 100): Number of individual observations for a particular object.

Column 8 (bytes 107 to 110): Comments. We have included in this column an acronym to indicate the nature of the star, when mentioned by the author. Acronyms are as follows:
CEPH: Cepheids.

ORB: An orbit has been computed. The radial velocity in Col. 6 is the systemic radial velocity.

SB1: Single lined binary.

SB2: Double lined binary.

EM: Emission lines. The radial velocity is derived from emission lines.

SB: Spectroscopic binary.

CV: Cataclysmic variable.

VAR: Star with variable radial velocity, according to the author.

LMC: Star belonging to the Large Magellanic Cloud.

STND: Radial velocity standard.

CONST: Star with constant radial velocity, according to the author.

SYMB: Symbiotic star.

BMP: Blue metal poor star.

VB: Visual binary.

Column 9 (bytes 115 to 120): Dispersion, resolving power or resolution. We have included in this column one of the above parameters. It is not possible to confuse the resolving power with any of the other two parameters because, in general, resolving power is a relatively large number compared with dispersion or resolution. As in the case of the dispersion or resolution, an ambiguity may arise, we have added an $\mathrm{R}$ to the number when it refers to the resolution. The acronym COR in this column means that the radial velocity was derived through cross correlation techniques (like Coravel, for example) while REL means a relative velocity, SEV means that more than one resolving power or resolution have been used, OP means objective prism observations, and IUE means that the radial 
Table 2. Sample page of the bibliographic catalogue

\begin{tabular}{|c|c|c|c|c|c|c|c|c|}
\hline Identification & 2000.0 coord. & $V$ & $\mathrm{ST}$ & RV & $N$ & Rem & $\mathrm{D} / \mathrm{RP} / \mathrm{R}$ & Reference \\
\hline G 7-2 & $0405.5+1103$ & 11.64 & K0 & -86.1 & 25 & & 22500 & AJ 107, 2240-1994 \\
\hline HD 25408 & $0405.9+6148$ & 7.60 & & Rvar... & 4 & & $\mathrm{SEV}$ & AJ 104, 1585-1992 \\
\hline G $160-45$ & $0406.5-1708$ & & M: & +77 & & & SEV & AJ 101, 1835-1991 \\
\hline HD 25642 & $0406.6+5021$ & 4.29 & A0IVn & & & & & PASP103, 1176-1991 \\
\hline HD 25425 & $0406.6+6531$ & 6.17 & $\mathrm{~A} 3 \mathrm{~m}$ & -3.8 & 2 & & 60000 & AAP $280,486-1993$ \\
\hline $\mathrm{BD}+53733$ & $0407.0+5411$ & 9.1 & F5 & -41.0 & 11 & & 22500 & AJ 107, 2240-1994 \\
\hline PPM - 14 & $0407.4+1114$ & & & & & & $\mathrm{COR}$ & AAP $285,943-1994$ \\
\hline HD 25893 & $0407.6+3805$ & 7.08 & G5 & & 12 & & $\mathrm{COR}$ & AAPS 88, 281-1991 \\
\hline HD 25893 & $0407.6+3805$ & 7.08 & G5 & +26.5 & 11 & & SEV & APJ 403, 708-1993 \\
\hline $\mathrm{BD}+37878 \mathrm{~A}$ & $0407.6+3805$ & 7.3 & $\mathrm{~K} 2$ & +26.9 & 8 & & 22500 & AJ 107, 2240-1994 \\
\hline HD 25998 & $0408.6+3803$ & 5.50 & F7V & & 7 & & COR & AAPS 88, 281-1991 \\
\hline HD 25940 & $0408.7+4743$ & 4.04 & $\mathrm{~B} 3 \mathrm{Ve}$ & +11.4 & 14 & & 39 & APJ 393, 666-1992 \\
\hline HD 26292 & $0409.7+0319$ & 6.5 & $\mathrm{~F} 2$ & -2.7 & 5 & & 20 & APJS 94, 677-1994 \\
\hline HD 26200 & $0410.4+3914$ & 6.99 & F0 & -53.4 & 5 & & 20 & APJS 94, 677-1994 \\
\hline HD 283420 & $0410.6+2522$ & 8.8 & F8 & +15.30 & 2 & & $\mathrm{COR}$ & AAP $255,130-1992$ \\
\hline G 221-29 & $0410.7+7422$ & 14.88 & DA: & -20.5 & 9 & & 22500 & AJ 107, 2240-1994 \\
\hline HD 26254 & $0411.0+4045$ & 8.3 & $\mathrm{~A} 2$ & +16 & 3 & & 80 & AAPS 94, 479-1992 \\
\hline HD 26285 & $0411.2+4145$ & 7.0 & K0 & +58 & 5 & & 80 & AAPS 94, 479-1992 \\
\hline $\mathrm{V}^{*} \mathrm{XY}$ Eri & $0411.3-1351$ & $?$ & & +221 & 3 & & SEV & AJ 108, 1016-1994 \\
\hline HD 26321 & $0411.4+4055$ & 7.2 & $\mathrm{~A} 2$ & +27 & 5 & & 80 & AAPS 94, 479-1992 \\
\hline HD 26310 & $0411.4+4152$ & 8.54 & F8 & +30 & 2 & & 80 & AAPS 94, 479-1992 \\
\hline HD 26584 & $0411.9-0850$ & 6.5 & G5 & +39.4 & 5 & & 20 & APJS 94, 677-1994 \\
\hline HD 284163 & $0411.9+2338$ & 9.35 & K0 & & & SB2 & 32000 & APJ 415, 150-1993 \\
\hline HD 26395 & $0412.3+4254$ & 8.2 & $\mathrm{~F} 4 \mathrm{~V} \ldots$ & +27 & 3 & & 80 & AAPS 94, 479-1992 \\
\hline HD 276122 & $0412.5+4200$ & 10.5 & K0 & +1.9 & 15 & & 22500 & AJ 107, 2240-1994 \\
\hline HD 26342 & $0412.8+5425$ & 7.8 & F0 & -22 & 3 & & 80 & AAPS 94, 479-1992 \\
\hline HD 26569 & $0414.0+4235$ & 8.0 & F0 & +14 & 3 & & 80 & AAPS 94, 479-1992 \\
\hline HD 283447 & $0414.2+2813$ & 10.5 & $\mathrm{~K} 2$ & +2.4 & 7 & & & APJ 432, 373-1994 \\
\hline $\mathrm{V}^{*} \mathrm{CW} \mathrm{Tau}$ & $0414.3+2811$ & 14.6 & $\mathrm{~K} 5 \mathrm{~V}: \mathrm{e} \ldots$ & +6 & 2 & & 10000 & AJ 103, 549-1992 \\
\hline $\mathrm{V}^{*} \mathrm{CW}$ Tau & $0414.3+2811$ & 14.6 & K5V:e... & +20.6 & 3 & & & APJS 93, 485-1994 \\
\hline HD 26512 & $0414.4+5431$ & 7.1 & $\mathrm{~A} 0$ & -10 & 6 & & 80 & AAPS $94,479-1992$ \\
\hline HD 284248 & $0414.6+2221$ & 9.2 & $\mathrm{~F} 2$ & +331 & & & SEV & AJ 101, 1835-1991 \\
\hline BD-06 855 & $0414.9-0538$ & 10.6 & $\mathrm{G}: \ldots$ & +296.2 & 22 & & 22500 & AJ 107, 2240-1994 \\
\hline HD 26702 & $0414.9+3733$ & 6.3 & G5 & -9.0 & 5 & & 20 & APJS 94, 677-1994 \\
\hline HD 26913 & $0415.4+0612$ & 6.93 & G5IV & -9.9 & & & COR & AJ 105, 226-1993 \\
\hline HD 26923 & $0415.5+0611$ & 6.30 & G0IV & -9.1 & & & COR & AJ 105, 226-1993 \\
\hline HD 26912 & $0415.5+0854$ & 4.2 & B3IV & +14.9 & 16 & & COR & AJ 101, 1495-1991 \\
\hline HD 26581 & $0415.5+583$ & 8.6 & K0 & +23.4 & 7 & & 22500 & AJ 107, 2240-1994 \\
\hline HD 26746 & $0415.6+4152$ & 7.8 & F0 & +59 & 5 & & 80 & AAPS 94, 479-1992 \\
\hline G $7-31$ & $0415.9+0754$ & 11.5 & $?$ & +29.5 & 5 & & 22500 & AJ 107, 2240-1994 \\
\hline HD 27290 & $0416.0-5129$ & 4.20 & F4III & +26.6 & 9 & & 1.9 & MN 267, 103-1994 \\
\hline G $175-25$ & $0416.2+5457$ & 13.4 & & -59.6 & 11 & & 22500 & AJ 107, 2240-1994 \\
\hline $\mathrm{BD}+50961$ & $0416.6+5038$ & 9.5 & Ce 3 & & & & $\mathrm{SEV}$ & AJ 104, 1585-1992 \\
\hline HD 26764 & $0416.7+533$ & 5.19 & $\mathrm{~A} 2 \mathrm{Vn}$ & -24 & 5 & & 80 & AAPS $94,479-1992$ \\
\hline $\mathrm{BD}+47965$ & $0417.3+4724$ & 9.93 & A3 & +5 & 6 & & SEV & AJ 108, 1016-1994 \\
\hline
\end{tabular}

velocities were derived from observations obtained with the International Ultraviolet Explorer.

Column 10 (bytes 123 - 139): Bibliographic reference. This column includes the bibliographic reference of the paper in which the radial velocities were published. Journal designations are abbreviated as shown in Table 1.

The stars in the catalogue are ordered by increasing right ascension. For a number of objects, we failed in obtaining an identification from the SIMBAD database. If the authors published coordinates for them, we used the designations and coordinates (precessed to J2000) provided in the reference. There are, however, 681 entries for which we have failed in obtaining an identification from SIMBAD and there are no coordinates published by the authors. These objects have been listed at the end of the catalogue in the last 681 lines. They will be merged with the rest of the identified objects as soon as we are able to find coordinates for them. 
Table 2 shows a page of the printed version of the catalogue.

Acknowledgements. This work was partially supported through a grant from the Consejo Nacional de Investigaciones Científicas y Técnicas, República Argentina PIP 4279.

\section{References}

Abt H., Biggs E., 1972, Bibliography of Stellar Radial Velocities. Latham Process Corp., New York
Barbier-Brossat M., Petit M., 1986, A\&A 65, 59

Barbier-Brossat M., Petit M., 1990, A\&AS 85, 885

Barbier-Brossat M., Petit M., Figon P., 1994, A\&AS 108, 603

Egret D., 1996, in SIMBAD User's Guide \& Reference Manual, Version 1.3, Centre de Données Astronomiques de Strasbourg, Strasbourg, France

Lortet M.C., Spite F., 1986, A\&AS 64, 329 